\title{
The Singularities of the Distance Function Near Convex Boundary Points
}

Paolo Albano

\begin{abstract}
In an open bounded set $\Omega$, we consider the distance function from $\partial \Omega$ associated to a Riemannian metric with $C^{1,1}$ coefficients. Assuming that $\Omega$ is convex near a boundary point $x_{0}$, we show that the distance function is differentiable at $x_{0}$ if and only if there exists the tangent space to $\partial \Omega$ at $x_{0}$. Furthermore, if the distance function is not differentiable at $x_{0}$ then there exists a Lipschitz continuous curve, with initial point at $x_{0}$, such that the distance function is not differentiable along such a curve.
\end{abstract}

Mathematics Subject Classification (2000). 26A27, 26A51,35F30.

Keywords. Eikonal equation, viscosity solutions, singularities, Dirichlet problem.

\section{Introduction and statement of the result}

Let $\Omega \subset \mathbb{R}^{n}$ be an open bounded set and consider the viscosity solution of the eikonal equation

$$
\left\{\begin{array}{l}
\langle A(x) \nabla d(x), \nabla d(x)\rangle=1 \quad \text { in } \Omega \\
d(x)=0 \text { for } \quad x \in \partial \Omega .
\end{array}\right.
$$

We assume that

$$
\bar{\Omega} \ni x \mapsto A(x) \text { is a matrix valued map of class } C^{1,1} \text { taking }
$$

values in the set of the positive definite matrices.

It is well-known that the above Dirichlet problem admits a unique (continuous) viscosity solution which is the distance function from $\partial \Omega$ with respect to the Riemannian metric $g_{x}(\xi, \xi)=\langle A(x) \xi, \xi\rangle$ (see [5]). We are interested in the structure of the singular set of the distance function:

$$
\Sigma(d):=\{x \in \bar{\Omega}: d \text { is not differentiable at } x\} .
$$

We recall that the points in $\Sigma(d) \cap \Omega$ have a simple geometrical interpretation: $x \in \Sigma(d) \cap \Omega$ if and only if there exists more than one geodesic from $x$ to $\partial \Omega$. 
Let $x_{0} \in \partial \Omega$ and assume that $\Omega$ is convex near $x_{0},{ }^{1}$ we consider the following problems:

A. characterize the property " $x_{0} \in \Sigma(d)$ " in terms of the geometry of $\partial \Omega$;

B. assuming that $x_{0} \in \Sigma(d)$, study the connected component of $\Sigma(d)$, inside $\Omega$, containing $x_{0}$.

Let us emphasize that, since we are interested in the structure of $\Sigma(d)$ near a boundary point, our convexity assumption is not a severe restriction. Indeed, because of a result of Motzkin (see [6]), the Euclidean distance function from a convex domain is differentiable in the exterior of the domain. In other words, the Euclidean distance function near "concave corners" does not have singularities.

The following result is our answer to question $A$.

Theorem 1.1. Let $\Omega$ be an open bounded set, assume (1.2) and let $x_{0} \in \partial \Omega$ be such that $\Omega \cap B_{\bar{\rho}}\left(x_{0}\right)$ is convex, for a suitable $\bar{\rho}>0$. Then, $x_{0} \in \Sigma(d)$ if and only if $\partial \Omega$ is not differentiable at $x_{0}$.

To address Question B, in the next result we show that the intersection of the connected component of $\Sigma(d)$ containing $x_{0}$ with $\Omega$ has dimension $\geq 1$.

Theorem 1.2. Let $\Omega$ be an open bounded set, assume (1.2) and let $x_{0} \in \partial \Omega$ be such that $\Omega \cap B_{\bar{\rho}}\left(x_{0}\right)$ is convex, for a suitable $\bar{\rho}>0$. Let $x_{0} \in \Sigma(d)$, then there exists a positive number $\sigma$ and a (non-constant) Lipschitz continuous curve $\gamma:[0, \sigma] \rightarrow$ $\Sigma(d)$ such that $\gamma(0)=x_{0}$ and $\gamma(s) \in \Omega$ for every $\left.\left.s \in\right] 0, \sigma\right]$.

\section{Preliminaries}

Let $u$ be a Lipschitz continuous function, the set of the reachable gradients of $u$ at $x$ is defined as

$$
D^{*} u(x)=\left\{\lim _{i \rightarrow \infty} \nabla u\left(x_{i}\right): x_{i} \rightarrow x, u \text { is differentiable at } x_{i}\right\} .
$$

Notice that, by the Rademacher theorem, $D^{*} u(x) \neq \emptyset$, for every $x$ in the domain of $u$. Let $D$ be an open convex set. We recall that the normal cone to $D$ at $x \in \partial D$ is defined as

$$
N_{D}(x)=\left\{p \in \mathbb{R}^{n}: \text { for every } y \in D,\langle p, y-x\rangle \leq 0\right\} .
$$

We say that $u: \bar{D} \rightarrow \mathbb{R}$ is semiconcave on $\bar{D}$ if there exists a positive constant $C$ such that

$$
\text { the function } \quad x \mapsto u(x)-C|x|^{2} \quad \text { is concave on } \quad \bar{D} \text {. }
$$

The constant $C$ above is a semiconcavity constant for $u$ on $\bar{D}$. We recall that the set valued map $x \mapsto D^{*} u(x)$ is upper-semicontinuous if $u$ is semiconcave on $\bar{D}$,

\footnotetext{
${ }^{1}$ Denoting by $B_{\bar{\rho}}\left(x_{0}\right)$ the Euclidean ball in $\mathbb{R}^{n}$ with center at $x_{0}$ and radius $\bar{\rho}>0$, we mean that there exists a positive number $\bar{\rho}$ such that $B_{\bar{\rho}}\left(x_{0}\right) \cap \bar{\Omega}$ is convex.
} 
i.e. if $\bar{D} \ni x_{i} \rightarrow x$ and $D^{*} u\left(x_{i}\right) \ni p_{i} \rightarrow p$, as $i \rightarrow \infty$, then $p \in D^{*} u(x)$. The superdifferential of $u$ at $x$ is

$$
D^{+} u(x)=\left\{p \in \mathbb{R}^{n}: \limsup _{\bar{D} \ni y \rightarrow x} \frac{u(y)-u(x)-\langle p, y-x\rangle}{|y-x|} \leq 0\right\} .
$$

If $u$ is semiconcave on $\bar{D}$ then, for every $x \in D, p \in D^{+} u(x)$ if and only if

$$
u(y) \leq u(x)+\langle p, y-x\rangle+C|x-y|^{2}, \quad \forall y \in D .
$$

Indeed, the fact that (2.3) implies that $p \in D^{+} u(x)$ is a direct consequence of the definition of the superdifferential (2.2), for a proof of the reverse implication see e.g. [3]. The constant $C$ in the above formula is a semiconcavity constant for $u$ on $\bar{D}$. A direct consequence of the above formula is that (2.3) holds for every $x \in \bar{D}$ and for every $p \in$ co $D^{*} d(x)$. Here "co" stands for the convex hull.

Remark 1. It is well-known that a semiconcave function $u$ is differentiable at $x \in D$ if and only if $D^{+} u(x)$ is a singleton. Moreover, for $x \in \bar{D}$ we have that co $D^{*} u(x) \subseteq D^{+} u(x)$ (equality holds iff $x$ belongs to the interior of $D$ ). See [3].

We complete this section recalling a result of [1] on the singularities of semiconcave functions.

Theorem 2.1. Let $u: D \rightarrow \mathbb{R}$ be a semiconcave function and let $x_{0} \in D$ be such that

$$
p_{0} \in \partial D^{+} u\left(x_{0}\right) \backslash D^{*} u\left(x_{0}\right),
$$

for a suitable $p_{0}$. Then for every $\theta \in N_{D^{+} u\left(x_{0}\right)}\left(p_{0}\right)$ there exist a positive number $\sigma$ and a Lipschitz continuous arc $\gamma:[0, \sigma] \rightarrow \Sigma(u)$ such that

$$
\gamma(0)=x_{0} \quad \text { and } \quad \lim _{s \rightarrow 0} \frac{\gamma(s)-x_{0}}{s}=-\theta .
$$

\subsection{Regularity of the distance function}

Assumption (1.2), more precisely the fact that the matrices $A(\cdot)$ are positive definite and the continuity of the map $x \mapsto A(x)$, yields that the Hamiltonian

$$
H(x, p)=\langle A(x) p, p\rangle-1
$$

is coercive w.r.t. the variables $p$ and the Lipschitz continuity of the viscosity solution of (1.1) follows by standard arguments. We remark that for every $x \in \bar{\Omega}$ we have that

$$
\langle A(x) p, p\rangle=1 \quad \text { for every } \quad p \in D^{*} d(x) .
$$

Arguing as in $[2]^{2}$ one can prove the following result

\footnotetext{
${ }^{2}$ In Theorem 3.1. of [2], it is shown that the minimum time function is (locally) semiconcave on the complement of the target set. But, as pointed out to me by Piermarco Cannarsa, the (local) semiconcavity constant is uniformly bounded, i.e. the semiconcavity holds up to the target.
} 
Theorem 2.2. Let $\Omega$ be an open bounded set, assume (1.2) and let $x_{0} \in \partial \Omega$ be such that $\Omega \cap B_{\bar{\rho}}\left(x_{0}\right)$ is convex, for some $\bar{\rho}>0$. Then there exists a positive number $\left.\left.\rho_{0} \in\right] 0, \bar{\rho}\right]$ such that the viscosity solution of the eikonal equation (1.1) is semiconcave on $\overline{\Omega \cap B_{\rho_{0}}\left(x_{0}\right)}$.

We remark that in the Euclidean case, $A(x) \equiv I d$, the distance function is concave near the point $x_{0}$. As shown in the next example, in the general case, one cannot expect concavity.

Example 1. Let $\Omega$ be a square and let $d_{e}$ be the Euclidean distance function from the boundary of $\Omega$. The graph of $d_{e}$ is a pyramid, consider one of the faces of the pyramid and denote by $T$ its projection on the base. Let $B \subset T$ a small ball away from the boundary of the triangle $T$ and let $\chi$ be a smooth cut-off function supported in $B$. In the interior of $T, d_{e}$ is differentiable and its gradient is constant, we denote by $v$ the gradient of $d_{e}$ on $T$. Take $\varepsilon$ small such that $v-\varepsilon \nabla \chi(x) \neq 0$ on $\Omega$. Then, the function $d(x)=d_{e}(x)-\varepsilon \chi(x)$ is the viscosity solution of the eikonal equation (1.1), with $A(x)=I /|v-\varepsilon \nabla \chi(x)|^{2}$, and it is clearly a not concave function.

\subsection{Generalized gradients of $d$ at boundary points}

In this section we describe the relationship between the normals to $\Omega$ at a boundary point $x$ and the generalized gradients of $d$ evaluated at $x$. For every point $x$ of differentiability for $\partial \Omega \cap B_{\rho_{0}}\left(x_{0}\right)$, as a consequence of the homogeneous Dirichlet boundary condition in (1.1) we have that

$$
D^{*} d(x)=\left\{\frac{-\nu(x)}{\sqrt{\langle A(x) \nu(x), \nu(x)\rangle}}\right\} .
$$

Here $\nu(x)$ is the unit outward normal to $\Omega$ at $x \in \partial \Omega$.

Proposition 2.1. Under the assumptions of Theorem 2.2, for every $x \in \partial \Omega \cap$ $B_{\rho_{0}}\left(x_{0}\right)$, we have

$$
-D^{+} d(x)=N_{\Omega}(x)
$$

and

$$
0 \notin \operatorname{co} D^{*} d(x) \text {. }
$$

Remark 2. The formula (2.7) can be also proved using the proximal calculus. We refer the reader interested in such an alternative proof to the monograph [4].

Proof. By Assumption (1.2) we have that there exist two positive constants $c_{1}<c_{2}$ such that

$$
c_{1} I \leq A(x) \leq c_{2} I \quad(\forall x \in \bar{\Omega}) .
$$

Denoting by $d_{e}$ the Euclidean distance function from $\partial \Omega$, we deduce that

$$
\frac{1}{\sqrt{c_{2}}} d_{e}(x) \leq d(x) \leq \frac{1}{\sqrt{c_{1}}} d_{e}(x) \quad(\forall x \in \bar{\Omega}) .
$$


Indeed, $d_{e} / \sqrt{c_{2}}$ and $d$ are a viscosity solution and supersolution (respectively) of the following Dirichlet problem

$$
c_{2}|\nabla u(x)|^{2}=1 \quad \text { in } \quad \Omega \quad u=0 \quad \text { on } \partial \Omega .
$$

Hence, the first inequality in (2.10) is a consequence of the comparison principle. The second inequality follows similarly considering the problem

$$
c_{1}|\nabla u(x)|^{2}=1 \quad \text { in } \quad \Omega \quad u=0 \quad \text { on } \partial \Omega .
$$

Let $x \in \partial \Omega \cap B_{\rho_{0}}\left(x_{0}\right)$, the definition of superdifferential, (2.2), yields that

$$
\frac{1}{\sqrt{c_{1}}} D^{+} d_{e}(x) \subset D^{+} d(x) \subset \frac{1}{\sqrt{c_{2}}} D^{+} d_{e}(x) .
$$

In order to complete the proof of (2.7) it is enough to show that

$$
-D^{+} d_{e}(x)=N_{\Omega}(x) .
$$

We remark that, near $x, d_{e}$ is a concave function hence $p \in D^{+} d_{e}(x)$ implies that, for $y \in \Omega$ near $x$,

$$
d_{e}(y) \leq d_{e}(x)+\langle p, y-x\rangle .
$$

Using the fact that the distance is a non-negative function and $d_{e}(x)=0$ we conclude that $-p \in N_{\Omega}(x)$. In other words

$$
D^{+} d_{e}(x) \subset-N_{\Omega}(x) .
$$

By the definition of superdifferential, (2.2), follows that

$$
D^{+} d_{e}(x)-N_{\Omega}(x) \subset D^{+} d_{e}(x) .
$$

We claim that (2.12) is a consequence of the inclusion (2.14) and (2.13). Indeed, assume by contradiction the existence of $p_{0} \in-N_{\Omega}(x) \backslash D^{+} d_{e}(x)$. Then, by the Hahn-Banach theorem, it follows that there exists a vector $\theta$ such that

$$
\left\langle p_{0}, \theta\right\rangle>0 \geq\langle p, \theta\rangle \quad \forall p \in D^{+} d_{e}(x) .
$$

Hence we get a contradiction taking in the above inequality $p$ of the form $q+t p_{0}$, with $q \in D^{+} d_{e}(x)$ and $t>0$, and sending $t \rightarrow+\infty$. This completes the proof of (2.12) and (2.7) follows.

Let us prove (2.8) arguing by contradiction assuming that

$$
0=\sum_{j=1}^{n+1} \lambda_{j} p_{j} \quad p_{j} \in D^{*} d(x), \quad \lambda_{j} \geq 0, \quad \sum_{j=1}^{n+1} \lambda_{j}=1 .
$$

Since $D^{*} d(x) \subset\{p:\langle A(x) p, p\rangle=1\}$ we can suppose that $\lambda_{1} \neq 0$. Then

$$
\lambda_{1} p_{1}+\left(1-\lambda_{1}\right) \sum_{j=2}^{n+1} \frac{\lambda_{j}}{\sum_{k=2}^{n+1} \lambda_{k}} p_{j}=0 .
$$

Recalling that the superdifferential is convex and that, at a boundary point, it is a conic set, we deduce that $\pm p_{1} \in D^{+} d(x)$. Then $\mathbb{R} p_{1} \subset N_{\Omega}(x)$ (due to (2.7)) which yields a contradiction: $\Omega \cap B_{\rho_{0}}\left(x_{0}\right)$ is open in $\mathbb{R}^{n}$ and $\Omega \cap B_{\rho_{0}}\left(x_{0}\right) \subset\left\{y \in \mathbb{R}^{n}\right.$ : $\left.\left\langle p_{1}, y-x\right\rangle=0\right\}$. Hence (2.8) follows. 


\section{Proof of Theorem 1.1}

The proof of the Theorem 1.1 is based on the following result concerning the existence of a semiconcave (local) extension preserving the set of the reachable gradients at the boundary points.

Proposition 3.1. Let $u$ be a semiconcave function defined in the convex set $\overline{\Omega \cap B_{\rho_{0}}\left(x_{0}\right)}$. Then for every $x \in \partial \Omega \cap B_{\rho_{0}}\left(x_{0}\right)$ there exist $\delta>0$ and a semiconcave function $\bar{u}: B_{\delta}(x) \rightarrow \mathbb{R}$ such that

$$
\bar{u}=u \quad \text { on } \quad \Omega \cap B_{\delta}(x),
$$

and

$$
D^{*} \bar{u}(y)=D^{*} u(y) \quad \forall y \in \partial \Omega \cap B_{\delta}(x) .
$$

Proof. Let $\chi \in C_{0}^{\infty}\left(\mathbb{R}^{n}\right)$ be a cut-off function with $\chi \equiv 1$ on $B_{\rho_{0} / 4}\left(x_{0}\right)$ and $\chi \equiv 0$ outside $B_{\rho_{0} / 2}\left(x_{0}\right)$. Then the function $\chi u$ is semiconcave in the convex set $\overline{\Omega \cap B_{\rho_{0}}\left(x_{0}\right)}$. For $x \in \mathbb{R}^{n}$, we define

$$
\bar{u}(x)=\inf _{y \in \overline{\Omega \cap B_{\rho_{0}}\left(x_{0}\right)}}\left[\chi(y) u(y)+\langle p, y-x\rangle+(C+1)|x-y|^{2}\right]
$$

(the constant $C$ is a semiconcavity constant for $\chi u$ on $\overline{\Omega \cap B_{\rho_{0}}\left(x_{0}\right)}$ ). We observe that, for $x \in \overline{\Omega \cap B_{\rho_{0}}\left(x_{0}\right)}, \bar{u}(x) \leq \chi(x) u(x)$ and $\bar{u}(x) \geq \chi(x) u(x)$ by (2.3). Hence, $\bar{u}=\chi u$ on $\overline{\Omega \cap B_{\rho_{0}}\left(x_{0}\right)}$. Moreover, since $\bar{u}$ is the infimum of a family of smooth functions with uniformly bounded second derivatives, it is a semiconcave function. It remains to check condition (3.1) and clearly it suffices to verify that $D^{*} \bar{u}(x) \subseteq$ $D^{*} u(x)$ for every $x \in \partial \Omega \cap B_{\rho_{0} / 4}\left(x_{0}\right)$.

Let $x_{n}$ be a sequence of differentiability points for $\bar{u}$ converging to $x \in \partial \Omega \cap$ $B_{\rho_{0} / 4}\left(x_{0}\right)$. We want to show that $\lim _{n \rightarrow \infty} \nabla \bar{u}\left(x_{n}\right) \in D^{*} u(x)$.

There exist a sequence of points $y_{n} \in \overline{\Omega \cap B_{\rho_{0}}\left(x_{0}\right)}$ and a sequence of vectors $p_{n} \in D^{*}(\chi u)\left(y_{n}\right)$ such that, for suitable $y_{0}$ and $p_{0}$,

(i) $\bar{u}\left(x_{n}\right)=\chi\left(y_{n}\right) u\left(y_{n}\right)+\left\langle p_{n}, x_{n}-y_{n}\right\rangle+(C+1)\left|x_{n}-y_{n}\right|^{2}$;

(ii) $y_{n} \rightarrow y_{0}$ and $p_{n} \rightarrow p_{0} \in D^{*}(\chi u)\left(y_{0}\right)$;

(the fact that $p_{0} \in D^{*}(\chi u)\left(y_{0}\right)$ is a consequence of the upper-semicontinuity of $\left.D^{*}(\chi u)\right)$. We have that $D^{+} \bar{u}\left(x_{n}\right)=\left\{\nabla \bar{u}\left(x_{n}\right)\right\}$ and, by the definition of the superdifferential, $p_{n}+2(C+1)\left(x_{n}-y_{n}\right) \in D^{+} \bar{u}\left(x_{n}\right)$. Hence

$$
\nabla \bar{u}\left(x_{n}\right)=p_{n}+2(C+1)\left(x_{n}-y_{n}\right) \rightarrow p_{0}+2(C+1)\left|x-y_{0}\right| \quad \text { as } \quad n \rightarrow \infty .
$$

Taking the limit, as $n \rightarrow \infty$, in the formula (i) above we find that

$$
\chi(x) u(x)=\chi\left(y_{0}\right) u\left(y_{0}\right)+\left\langle p_{0}, x-y_{0}\right\rangle+(C+1)\left|x-y_{0}\right|^{2} .
$$

Using the above equation the fact that $p_{0} \in D^{*}(\chi u)\left(y_{0}\right)$ and Formula (2.3) we deduce that $\left|x-y_{0}\right|=0$. Hence $\lim _{n \rightarrow \infty} \nabla \bar{u}\left(x_{n}\right)=p_{0}$ and, recalling that $\left.\chi\right|_{B_{\rho_{0} / 4}\left(x_{0}\right)}=$ $1,(3.1)$ follows. 
Now, let $x_{0}$ be a point of differentiability for $\partial \Omega$. Applying the previous result to the distance function $d$, we obtain that there exists a semiconcave extension $\bar{d}$, defined on a full neighbourood of $x_{0}$, such that

$$
D^{+} \bar{d}\left(x_{0}\right)=\operatorname{co} D^{*} \bar{d}\left(x_{0}\right) .
$$

By construction, $D^{*} \bar{d}\left(x_{0}\right)=D^{*} d\left(x_{0}\right)$ and $D^{*} d\left(x_{0}\right)$ is a singleton by (2.6). Hence $D^{+} \bar{d}\left(x_{0}\right)$ is a singleton, i.e. it is differentiable at $x_{0}$.

Finally, if $x_{0} \in \Sigma(d)$ then (2.7) yields that $\operatorname{dim} N_{\Omega}\left(x_{0}\right)>1$, i.e. $\partial \Omega$ is not differentiable at $x_{0}$. This completes the proof of Theorem 1.1.

\section{Proof of Theorem 1.2}

In this section we apply Theorem 2.1 to the function $\bar{d}$. We begin by checking that Assumption (2.4) is fulfilled. Let $x_{0} \in \Sigma(d)$ and consider the following problem: minimize the (strictly convex) function $p \mapsto\left\langle A\left(x_{0}\right) p, p\right\rangle$ over the set $D^{+} \bar{d}\left(x_{0}\right)$. Denote by $p_{0} \in D^{+} \bar{d}\left(x_{0}\right)$ the minimizer of the above function, i.e.

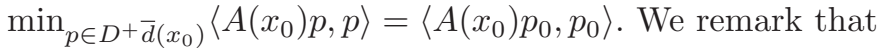

$$
\left\langle A\left(x_{0}\right) p_{0}, p\right\rangle \geq\left\langle A\left(x_{0}\right) p_{0}, p_{0}\right\rangle>0 \quad \forall p \in D^{+} \bar{d}\left(x_{0}\right),
$$

in the last inequality we used the fact that $0 \notin \operatorname{co} D^{*} d\left(x_{0}\right)=D^{+} \bar{d}\left(x_{0}\right)$. Notice that the strict convexity of the function $p \mapsto\left\langle A\left(x_{0}\right) p, p\right\rangle$, the fact that $\left\langle A\left(x_{0}\right) p, p\right\rangle=1$ on $D^{*} \bar{d}\left(x_{0}\right)$, and the fact that since $x_{0} \in \Sigma(d)$ then $D^{*} d\left(x_{0}\right)=D^{*} \bar{d}\left(x_{0}\right)$ contains at least two distinct elements yield that

$$
p_{0} \in D^{+} \bar{d}\left(x_{0}\right) \backslash D^{*} \bar{d}\left(x_{0}\right) .
$$

Define

$$
\theta:=-A\left(x_{0}\right) p_{0} .
$$

Observe that $\theta \in N_{D^{+} \bar{d}\left(x_{0}\right)}\left(p_{0}\right)$, by (4.3), and, using (4.3) once more, we deduce that for every $h>0 p_{0}+h \theta \notin D^{+} \bar{d}\left(x_{0}\right)$, i.e. $p_{0} \in \partial D^{+} \bar{d}\left(x_{0}\right)$ and (2.4) holds. Now, applying Theorem 2.1 to the function $\bar{d}$ we find a Lipschitz continuous arc $\gamma:[0, \sigma] \rightarrow \Sigma(\bar{d})$ such that

$$
\gamma(0)=x_{0} \quad \text { and } \quad \lim _{s \rightarrow 0} \frac{\gamma(s)-x_{0}}{s}=-\theta .
$$

It remains to show that, possibly taking a smaller $\sigma, \gamma(s) \in \Omega$, for every $s \in] 0, \sigma]$. We remark that, due to $(4.3),\langle\theta, p\rangle<0$ for every $p \in D^{+} \bar{d}\left(x_{0}\right)$, hence

$$
\langle\theta, n\rangle>0 \quad \forall n \in N_{\Omega}\left(x_{0}\right)=\mathbb{R}^{-} D^{+} \bar{d}\left(x_{0}\right) .
$$

In order to complete the proof it suffices to show that there are no sequences of points $x_{n} \in \mathbb{R}^{n} \backslash \Omega$ such that

$$
\mathbb{R}^{n} \backslash \Omega \ni x_{n} \rightarrow x_{0} \quad \text { and } \quad \lim _{n \rightarrow \infty} \frac{x_{n}-x_{0}}{\left|x_{n}-x_{0}\right|}=-\frac{\theta}{|\theta|} .
$$

We argue by contradiction. Consider two cases: 
(a) there exists a sequence $x_{n} \notin \bar{\Omega}$ such that (4.5) holds;

(b) there exists a sequence $x_{n} \in \partial \Omega$ such that (4.5) holds.

Case (a): let $y_{n}$ be the (Euclidean) projection of $x_{n}$ on $\partial \Omega$, i.e. $x_{n}-y_{n} \in N_{\Omega}\left(y_{n}\right)$. Then

$$
0 \geq\left\langle x_{n}-y_{n}, x_{0}-y_{n}\right\rangle=\left\langle x_{n}-y_{n}, x_{0}-x_{n}\right\rangle+\left|x_{n}-y_{n}\right|^{2}
$$

and we deduce that

$$
0 \geq\left\langle x_{n}-y_{n}, x_{0}-x_{n}\right\rangle .
$$

Dividing both sides of the above inequality by $\left|x_{n}-y_{n}\right|\left|x_{0}-x_{n}\right|$ and taking the limit as $n \rightarrow \infty$ we obtain that

$$
0 \geq\left\langle\lim _{n \rightarrow \infty} \frac{x_{n}-y_{n}}{\left|x_{n}-y_{n}\right|},-\frac{\theta}{|\theta|}\right\rangle \quad \text { and } \quad \lim _{n \rightarrow \infty} \frac{x_{n}-y_{n}}{\left|x_{n}-y_{n}\right|} \in N_{\Omega}\left(x_{0}\right)
$$

which contradicts (4.4).

Case (b): let $\nu_{n} \in N_{\Omega}\left(x_{n}\right)$. We can suppose that $\nu_{n} \rightarrow \nu_{\infty} \in N_{\Omega}\left(x_{0}\right)$. Hence taking the limit, as $n \rightarrow \infty$, in the inequality

$$
\left\langle\nu_{n}, \frac{x_{0}-x_{n}}{\left|x_{0}-x_{n}\right|}\right\rangle \leq 0
$$

we deduce that

$$
\left\langle\nu_{\infty}, \frac{\theta}{|\theta|}\right\rangle \geq 0 \quad\left(\text { for } \quad \nu_{\infty} \in N_{\Omega}\left(x_{0}\right)\right),
$$

which contradicts (4.4). This completes our proof.

\section{References}

[1] P. Albano and P. Cannarsa, Structural properties of singularities of semiconcave functions, Ann. Scuola Norm. Sup. Pisa Cl. Sci. 28 (1999), 719-740.

[2] P. Cannarsa and C. Sinestrari, Convexity properties of the minimum time function, Calc. Var. Partial Differential Equations 3 (1995), 273-298.

[3] P. Cannarsa and C. Sinestrari, Semiconcave functions, Hamilton-Jacobi equations, and optimal control. Progress in Nonlinear Differential Equations and their Applications, 58. Birkhäuser Boston, 2004.

[4] F. H. Clarke, Yu. S. Ledyaev, R. J. Stern and P. R. Wolenski, Nonsmooth analysis and control theory. Graduate Texts in Mathematics, 178. Springer Verlag, New York, 1998.

[5] P. L. Lions, Generalized solutions of Hamilton-Jacobi equations. Research Notes in Mathematics, 69. Pitman, Boston, Mass.-London, 1982.

[6] T. Motzkin, Sur quelques propriétés caractéristiques des ensembles convexes, Atti Accad. Naz. Lincei, Rend., VI. Ser. 21 (1935), 562-567. 
Paolo Albano

Dipartimento di Matematica

Università di Bologna

Piazzadi Porta San Donato 5

40127 Bologna

Italy

e-mail: albano@dm.unibo.it

Received: 3 April 2008.

Accepted: 19 November 2008. 